\title{
AN ALPHA PARTICLE DIAGNOSTIC BASED ON MEASUREMENTS OF LOWER HYBRID WAVE FLUCTUATIONS
}

\author{
K. L. Wong \\ Princeton Plasma Physics Laboratory, Princeton University \\ Princeton, N.J. 08543, USA
}

\begin{abstract}
It is shown that the one-dimensional alpha particle velocity distributi $n$ function can be determined from the fluctuation-dissipation theorem based on measurements of lower hybrid wave fluctuations in an equilibrium plasma. This method uses collective Thomson scattering data with large signal-to-noise ratio, but it is applicable only when the alpha particles have an isotropic velocity distribution.
\end{abstract}

\section{DISCLAIMER}

Thrs report wis prepared as an account of work sporixored by an agency of the Innited States Government Neither the United States Guverniment nor anty agency thereof. not any uf their employees. makes any warranty, express or implted, ur assumes any legal labilaty or responss. bility for the accuracy. Completeness. ur usefulness af any information. upparatus. product. or process diselused, or represents that its use would not inftinge privalcly owned rights Relerence herein to any specific commercial product. process, ar service by irade name. tritdernark. munufacturer, or otherwise does not necessarily cunstutute or imply its endorsement. recom. mendation. w fivoring by the Unuted States Governinent of any agency theteof The vews and opinions of authors expressed hereis do not necessafily state or reflect those of the Uniled Slates Guvernment of any juensy thereaf 
As present tokamak parameters approach the reactor regime, the interest in fusion research stants to focus on alpha particle behavior [1] in ignited plasmas. While new machines are being designed for this purpose, it is noticed that the diagnostic for alpha particles is one major item which needs to be developed. In order to study alpha particle physics, one would like to measure the alpha particle distribution function $f_{\alpha}(\vec{r}, \vec{v}, t)$ with good resolution in space, velocity, and time. Many schemes have been proposed recently, and each one has its own difficulties [2]. One method that is being seriously investigated is collective Thomson scattering [3]. This process has a very small cross section that is characterized by the classical electron radius $\left(r_{\theta}=e^{2} / \mathrm{mc}^{2}=2.82 \times 10^{-13} \mathrm{~cm}\right)$. Therefore, high incident beam power is necessary. A $100 \mathrm{MW} \mathrm{CO}_{2}$ laser $(10.6 \mu \mathrm{m})$ has been proposed. Because of the short wavelength, the scattering angle is very small $\left(\theta \leq 1^{\circ}\right)$, which gives rise to very poor spatial resolution. Such a high power laser must be operated in a pulsed mode and can only take measurements at one point in time. The power requirement is substantially reduced when one uses $1-2 \mathrm{~mm}$ microwave sources. A few hundred kilowatts of microwave power would be sufficient [4]. At these long wavelengths, wave retraction inside the plasma will introduce complications, but this is not an unsurmountable difficulty. The scattering angle will be large enough to provide good spatial resolution. Calculations [5,6] of the spectral function $S(\vec{k}, \omega)$ show that if $k_{z} / k_{\perp}\left(k_{z}\right.$ is the component of $\vec{k}$ parallel to the magnetic field and $k_{n}$ is the component perpendicular to the magnetic field) is not too small, the alpha particle feature in $\mathrm{S}$ can become dominant and the onedimensional alpha panticle distribution function can be obtained from the frequency spectrum of $S(\vec{k}, \omega)$ at one fixed value of $\vec{k}$. Large enhancement of $S(\vec{k}, \omega)$ was noticed due to lower hybrid waves at small values of $\mathrm{k}_{\mathrm{z}} / \mathrm{k}_{\perp}$. The scattering signal can be three orders of magnitude higher [7]. This large signal is discarded because of the difficulty in data interpretation. In this paper, we investigate the possibility of using this large signal to extract information about the alpha particle distribution function. If successful, we may be able to increase the signal-to-noise ratio by three orders of magnitude, or reduce the incident power requirement by three orders of magnitude so that steady-state operation of the diagnostic can be easily realized. 
It has been shown that lower hybrid waves can be strongly damped by energetic alpha particles in a tckamak reactor [8]. Therefore, the fluctuation level of lower hybrid waves is sensitive to the velocity distribution function of the alpha particles ${ }_{\alpha}^{f}(\vec{v})$. A lower hybrid wave is an electrostatic wave in the frequency range $\omega_{\mathrm{ci}} \ll \omega \ll \omega_{c e}$, and it is a common practice to assume magnetized electrons and unmagnetized ions for the wave dispersion relation. Suppose the plasma is at equilibrium. The spectral power density of electrostatic fluctuations in the plasma can be estimated from the fluctuation-dissipation theorem [9] as follows:

$$
\begin{aligned}
P(\vec{k}, \omega) & =\frac{n_{\theta} e^{2}}{k^{2} \varepsilon_{0}^{2}\left|K_{L}(\vec{k}, \omega)\right|^{2}} \int d^{3} v \sum_{n} J_{n}\left(\frac{k, v_{\perp}}{\omega_{c \theta}}\right) \delta\left(\omega-n \omega_{c \theta}-k_{z} v_{z}\right) f_{\theta}(\vec{v}) \\
& +\frac{n_{i} e_{i}^{2}}{k^{2} \varepsilon_{0}^{2}\left|K_{L}(\vec{k}, \omega)\right|^{2}} \int d^{3} v f_{i}(\vec{v}) \delta(\omega-\vec{k} \cdot \vec{v}) \\
& +\frac{n_{\alpha} e_{\alpha}^{2}}{k^{2} \varepsilon_{0}^{2}\left|K_{L}(\vec{k}, \omega)\right|^{2}} \int d^{3} v f_{\alpha}(\vec{v}) \delta(\omega-\vec{k} \cdot \vec{v})
\end{aligned}
$$

where $\begin{array}{ll}n_{j} & =\text { particle density of the } j \text {-species } \\ e_{j} & =\text { electric charge of the } j \text {-species } \\ \omega_{c e}= & \text { electron cyclotron frequency } \\ k_{L} & =\vec{k} \cdot \vec{k} \cdot \vec{k} k{ }^{2} \text { is the longitudinal dielectric function }(\vec{K} \text { is } \\ & \text { the dielectric tensor). }\end{array}$

The magnetic field is alor? the $\hat{z}$-axis. The three terms in Eq. (1) correspond to those in the spectral function $[5,6] \mathrm{S}(\vec{k}, \omega)$ which determines the electron density fluctuation $\tilde{n}_{a}(\vec{k}, \omega)$. From $\bar{n}_{e}(\vec{k}, \omega)$, one can easily calculate the electric 
fluctuation $\vec{E}(\vec{k}, \omega)$ and $P(\vec{k}, \omega)$. Hence, $P(\vec{k}, \omega)$ is treated as a quantity measured in the experiment.

Lower hybrid waves with wave number $\vec{k}$ and wave frequency $\omega$ are normal modes of the plasma. The real part of $K_{L}(\vec{k}, \omega)$ vanishes, which causes signiticant enhancement in $P(\vec{k}, \omega)$ and $S(\vec{k}, \omega)$. When $\omega \gg \omega_{p i},\left(\omega_{p i}\right.$ is the ion plasma frequency), the wave dispersion relation is:

$$
\frac{k_{z}^{2}}{k^{2}}=\frac{(1)^{2}}{\omega_{p \theta}^{2}}\left(1+\frac{\omega_{p \theta}^{2}}{\omega_{s \theta}^{2}}\right) \ll 1
$$

When $\omega \sim \omega_{p i}$, the ion dynamics becomes important. If one neglects finite Larmor radius effects, there is a lower hybrid resonance $\left(k_{\perp} \rightarrow \infty\right)$ when

$$
\omega=\omega_{L H}=\left(\frac{1}{\omega_{c i}^{2}+\omega_{p}^{2}}+\frac{1}{\omega_{c i} \omega_{c \theta}}\right)^{-1 / 2}
$$

This resonance is removed by ion linite Larmor radius effects $[10,11]$. Nevertheless, when $\omega \sim \omega_{\mathrm{pi}}, \mathrm{k}_{\mathrm{z}} / \mathrm{k}$ is even smaller than that given in Eq. (2). Therefore, $P(\vec{k}, \omega)$ and $S(\vec{k}, \omega)$ are significantly larger at small values of $k_{z} / k$ in the lower hybrid frequency range, as was noted in previous calculations $[5,6]$. Since we know that lower hybrid waves can be absorbed by energetic alpha particles, the imaginary part of $K_{L}(\vec{k}, \omega)$ contains information on the alpha particle velocity distribution iunction $\mathrm{f}_{\alpha}(\overrightarrow{\mathrm{v}})$. We will show that under certain conditions, the large values of $P(\vec{k}, \omega)$ or $S(\vec{k}, \omega)$ due to lower hybrid waves can be used to obtain information on $f_{\alpha}(\vec{v})$. 
Let $v$, and $v_{\perp}$ be the velocity components parallel and perpendicular to $\vec{k}$, respectively. The one-dimensional distribution function [12] is defined as:

$$
F(u)=\int f_{\alpha}(\vec{v}) \delta\left(u-\frac{\vec{k} \cdot \vec{v}}{k}\right) d^{3} v=\int_{-\infty}^{\infty} d v_{\|} \delta\left(u-v_{n}\right) \int_{0}^{-} f_{\alpha}(\vec{v}) 2 \pi v_{\perp} d v_{\perp}
$$

The alpha particle contribution to $K_{L}(\vec{k}, \omega)$ is:

$$
\chi_{\alpha}=-\frac{\omega_{\rho \alpha}^{2}}{k^{2}} \int \frac{d F / d u}{L-(\omega / k)} d u=-\frac{\omega_{\rho \alpha}^{2}}{k^{2}}\left[P \int_{\infty}^{\infty} \frac{d F / d u}{u-(\omega / k)} d u+\left.i \pi \frac{d F}{d u}\right|_{\omega / k}\right] \equiv \chi_{\alpha R}+i \chi_{\alpha u}
$$

In the above expression, $L$ denotes the Landau contour and $P$ denotes the principal value of the integral. In the case of $\omega>>\omega_{p i}$, we have

$$
\begin{aligned}
K_{\perp}(\vec{k}, \omega) & =\frac{k_{\perp}^{2}}{k^{2}}\left(1+\frac{\omega_{p \theta}^{2}}{\omega_{c \theta}^{2}}-\frac{\omega_{p i}^{2}}{\omega^{2}}\right)+\frac{k_{z}^{2}}{k^{2}}\left(1-\frac{\omega_{p \theta}^{2}}{\omega^{2}}\right)+\chi_{\alpha R}+\frac{i \sqrt{\pi}}{k^{2} \lambda_{d \theta}^{2}} \frac{\omega}{k_{z} v_{z}} \exp \left(-\frac{\omega^{2}}{k_{z}^{2} v_{\theta}^{2}}\right) \\
& +\frac{i \sqrt{\pi}}{k^{2} \lambda_{d i}^{2}} \frac{\omega}{k v_{i}} \exp \left(-\frac{\omega^{2}}{k^{2} v_{i}^{2}}\right)-\left.i \pi \frac{\omega_{p \alpha}^{2}}{k^{2}} \frac{d F}{d u}\right|_{\omega / k}=K_{R}(\vec{k}, \omega)+i K_{I}(\vec{k}, \omega)
\end{aligned}
$$

We have assumed Maxwellian distribution for the plasma electrons ( $v_{\theta}=$ electron thermal velocity) and ions $\left(v_{i}=\right.$ ion thermal velocity). $\lambda_{d j}=\omega_{p j} / v_{j}$ is the Debye length of the j-species. The three terms in $K_{r}(\vec{k}, \omega)$ correspond to wave damping due to electrons, ions, and alpha particles. The spectral power density of lower hytrid waves, which have the dispersion relation $\varsigma_{\mathrm{F}}(\vec{k}, \omega)=0$, can be calculated from Eq. (1): 


$$
\begin{aligned}
P(\vec{k}, \omega) & =\frac{n_{\theta} e^{2}}{k_{z} k^{2} \varepsilon_{0}^{2}} \frac{\left.\exp (-\omega)^{2} / k_{z}^{2} v_{e}^{2}\right)}{\left.\sqrt{\pi} v_{e}\left|K_{1}(\vec{k}, \omega)\right|\right|^{2}}+\frac{n_{i} e_{i}^{2}}{k^{3} \varepsilon_{0}^{2}} \frac{\exp \left(-\omega^{2} k^{2} v_{i}^{2}\right)}{\sqrt{\pi} v_{i}\left|K_{I}(\vec{k}, \omega)\right|^{2}} \\
& +\frac{n_{\alpha} e_{\alpha}^{2}}{k^{3} \varepsilon_{0}^{2}} \frac{F(\omega)||_{\omega / k}}{\left|K_{1}(\vec{k}, \omega)\right|^{2}}
\end{aligned}
$$

In the parameter regime where $\omega / k_{z} v_{e} \gg 1$ and $\omega / k v_{i} \gg 1$, the electron and ion contributions to $P\left(\vec{k}_{0} \omega\right)$ are exponentially small, and the alpha particle contribution can be dominant. Let us assume that the alpha particles have a slowing-down distribution, namely,

$$
\begin{aligned}
f_{\alpha}(\vec{v}) & =\frac{F_{0}}{v^{3}+v_{c}^{3}} \equiv \frac{F_{0}}{\left(v_{1}^{2}+v_{\| l}^{2}\right)^{3 / 2}+v_{c}^{3}} \text {, when } 0 \leq v \leq v_{\alpha} \\
& =0 \text {, otherwise } \\
v_{\alpha} & =v \text { slocity of } 3.5 \text { Mev alpha particles } \\
v_{c} & =\left(\frac{3 \sqrt{\pi} m_{\theta} z_{i}}{4 m_{\alpha}}\right)^{1 / 3} v_{\theta}, z_{1}=\frac{n_{i} z_{i}^{2} m_{a}}{n_{\theta} m_{i}}+\frac{n_{\alpha} z_{\alpha}^{2}}{n_{\theta}}
\end{aligned}
$$

$v_{C}$ is the critical alpha particle velocity at which electron drag equals ion drag [13], and $F_{0}=3 /\left[4 \pi \ln \left(1+v_{x}{ }^{3} / v_{c}^{3}\right)\right]$ is the normalization constant. The onedimensional velocity distribution function can then be calculated from Eq. (4), which yields: 


$$
\begin{aligned}
F(u)= & -\frac{2 \pi}{3} \frac{F_{0}}{v_{c}}\left\{\frac{3}{2} \ln \left[\frac{\left(v_{\alpha}+v_{c}\right) /\left(v_{\alpha}^{3}+v_{c}^{3}\right)^{1 / 3}}{\left(l|u|+v_{c}\right) r\left(|u|^{3}+v_{c}^{3}\right)^{1 / 3}}\right]+\sqrt{3} \tan ^{-1}\left(\frac{\sqrt{3} v_{\alpha}}{v_{\alpha}-2 v_{c}}\right)\right. \\
& \left.-\sqrt{3} \tan ^{-1}\left(\frac{\sqrt{3}|u|}{|u|-2 v_{c}}\right)\right\}
\end{aligned}
$$

It should be noted here that Eq. (8) is different from previously published results $[6,14]$. For the benefit of those who are interested in checking the algebra, the detailed steps are outlined in the Appendix. The spectral power density can be obtained by substitution of Eq. (B) into Eq. (7).

The purpose of the alpha particle diagnostic is to measure $f_{\alpha}(\vec{v})$ or its related quantities, for example, the aipha particle density, or other moments of $f_{\alpha}(\vec{v})$. It has been shown $[5,6]$ that the one-dimensional alpha particle distribution function $F(u)$ can be obtained from the frequency spectrum of $S(\vec{k}, \omega)$ at one fixed value of $\vec{k}$ provided that $k_{z} / k$ is large enough to avoid signals from lower hybrid wave fluctuations, which are much larger signals at small values of $k_{z} / k$. Here, we will show that these large signals can be used to obtain the same information provided that $f_{\alpha}(\vec{v})$ is isotropic. In a large reactor, $f_{\alpha}(\vec{v})$ is expected to be isotropic in the region where prompt orbit loss is not important, and the one-dimensional distribution function $F(u)$ would be independent of the direction of $\vec{k}$. Let us consider a schematic setup as shown in Fig. 1 . The scattering angle $\theta$ is kept fixed while the scattered wave number $\vec{k}_{s}$ moves on a conical surface. The magnitude of $\vec{k}$ is kept fixed; this is realized experimentally by placing detectors on the circle shown in Fig. 1. Signals from different detectors corresporid to different values of $k_{z} / k$ while $k$ is held fixed. With these values of $k_{z}$ and $k$, the lower hybrid wave frequency $\omega$ can be calculated from the dispersion relation $K_{R}(\vec{k}, \omega)=0$, and the signal from the detector measures the value of $P(\vec{k}, \omega)$ at a particular value of $u=\omega / k$. The array of detectors on the circle shown in Fig. 1 will determine $P(\vec{k}, \omega)$ over a range of $u$. Let us put $P(u)=P(\vec{k}, \omega)$ as a known function of $u$, then the one. dimensional distribution function $F(U)$ can be determined from Eq. (7). For the 
general case in which lower hybrid wave damping by the bulk plasma is not entirely negligible, we have

$$
K_{I}(\vec{k}, \omega)=\frac{\sqrt{\pi}}{k^{2} \lambda_{d \theta}^{2}} \frac{\omega}{k_{z} v_{\theta}} \exp \left(-\frac{\omega^{2}}{k_{z}^{2} v_{\theta}^{2}}\right)+\frac{\sqrt{\pi}}{k^{2} \lambda_{d i}^{2}} \frac{\omega}{k v_{i}} \exp \left(-\frac{\omega^{2}}{k^{2} v_{i}^{2}}\right)-\pi \frac{\omega_{p \alpha}^{2}}{k^{2}} \frac{d F}{d u} .
$$

From the dispersion relation $K_{F}(\vec{k}, \omega)=0, \omega / K_{Z}$ and $k_{z}$ can be expressed in terms of $\omega / k \equiv u$, and we can write $K_{L}(\vec{k}, \omega)$ in the following form:

$$
K_{I}(\vec{k}, \omega)=L_{1}(u)-\pi \frac{\omega_{p \alpha}^{2}}{k^{2}} \frac{d F}{d u}
$$

By the same token, Eq. (7) can be written as

$$
P(u)=L_{2}(u)+\frac{n_{\alpha} e_{\alpha}^{2}}{k^{3} \varepsilon_{0}^{2}} \frac{F(u)}{\left[L_{1}(u)-\pi\left(\omega_{\rho 0}^{2} / k^{2}\right)(d F / d u)\right]^{2}}
$$

or

$$
\pi \frac{\omega_{\rho \alpha}^{2}}{k^{2}} \frac{d F}{d u}=L_{1}(u)-\left[\frac{n_{\alpha} e_{\alpha}^{2}}{k^{3} \varepsilon_{0}^{2}} \frac{F(u)}{P(u)-L_{2}(u)}\right]^{1 / 2}
$$

Since $P(u), L_{1}(u)$, and $L_{2}(u)$ are known functions of $u, F(u)$ can be solved by numerical methods.

A.s an illustration of the above scheme. let us consider the simple case where $\omega / k_{2} v_{e}>1$ and $\omega / k v_{i} \gg 1$ such that Landau damping of the bulk plasma is negligible, then $L_{1}(u) \simeq 0, L_{2}(u) \simeq 0$, and Eq. $(10)$ is reduced to 
$\pi \frac{\omega_{p \alpha}^{2}}{k^{2}} \frac{d F}{d u}=-\left[\frac{n_{\alpha} e_{\alpha}^{2}}{k^{3} \varepsilon_{0}^{2}} \frac{F(u)}{P(u)}\right]^{1 / 2}$

Equation (11) is integrable, and the solution is:

$$
F(u)=\frac{C_{1}}{4}\left[\int \frac{d u}{[P(u)]^{1 / 2}}+C_{2}\right]^{2},
$$

where $C_{1}=k m_{\alpha}^{2} / \pi^{2} n_{\alpha} e_{\alpha}^{2}$, and $C_{2}$ is the integration constant determined from the condition that $F(u)=0$ for $u \geq v_{\alpha}$. Normalization of $F(u)$ will determine $C_{1}$ and $n_{\alpha}$.

In summary, we have discovered a method to determine the one-dimensional alpha particle distribution function from collective scattering of lower hybrid wave fluctuations in the plasma. This method has the potential of substantial improvement in the signal-to-noise ratio, but it is applicable only when $f_{\alpha}(\vec{v})$ is isotopic, and it involves more complicated data analysis. Since this method employs the natural fluctuation of lower hybrid waves in the plasma interior, it should be better than the method of measuring the damping of externally launched lower hybrid waves [15] whose power spectrum can be aitered by density fluctuations along the path of wave propagation. The spatial resolution should be comparable to the absorption length of lower hybrid waves, which is much less than the minor radius for tokamak plasmas close to thermonuclear ignition [8].

\section{ACKNOWLEDGMENTS}

The author would like to thank Dr. Kenneth Young for the suggestion of this problem, and Dr. John Krommes for a very helpful discussion on the fluctuationdissipation theorem. Comments given by Dr. N. Bretz and Dr. R. Goldzion were also very helpful to this manuscript. This work is supported by United States Department of Energy Contract No. DE-AC02-76-CHO3O73. 


\section{APPENDIX}

By putting the slow-down distribution function into Eq. (4), we obtain

$$
\begin{aligned}
F(u) & =\int_{-\infty}^{\infty} d v_{\|} \delta\left(u-v_{\| i}\right) \int_{0}^{v_{\alpha}^{2} \cdot v_{\|}^{2}} \frac{\pi F_{0} d\left(v_{\perp}^{2}\right)}{\left(v_{\perp}^{2}+v_{\|}^{2}\right)^{3 / 2}+v_{c}^{3}} \\
& =\int_{-\infty}^{\infty} d v_{\|} \delta\left(u-v_{\|}\right) \cdot\left(\frac{2}{3} \pi F_{0}\right) \mid \int_{\mid \alpha_{\|}}^{v^{3}+v_{c}^{3}+v_{c}^{3}} \frac{d y}{y\left(y-v_{c}^{3}\right)^{1 / 3}},
\end{aligned}
$$

where $y=\left(v_{\perp}^{2}+v_{\|}^{2}\right)^{3 / 2}+v_{c}^{2}$. From the integral table by Gradshteyn and Ryzhik [16], the integral over $y$ can be carried out, and yield

$$
\begin{aligned}
& F(u)=\int_{-\infty}^{\infty} d v_{\|} \delta\left(u-v_{\|}\right)\left(-\frac{2 \pi}{3} F_{o}\right) \frac{1}{v_{c}}\left\{\frac{3}{2} \ln \left[\frac{\left(y-v_{c}^{3}\right)^{1 / 3}+v_{c}}{y^{1 / 3}}\right]\right. \\
& \left.+\sqrt{3} \tan ^{-1}\left[\frac{\sqrt{3}\left(y-v_{c}^{3}\right)^{1 / 3}}{\left(y-v_{c}^{3}\right)^{1 / 3}-2 v_{c}}\right]\right\}\left.\right|^{v_{a}^{3}+v_{c}^{3}} \\
& =-\frac{2 \pi}{3} \frac{F_{0}}{v_{c}}\left\{\frac{3}{2} \ln \left[\frac{\left(v_{\alpha}+v_{c}\right) /\left(v_{\alpha}^{3}+v_{c}^{3}\right)^{1 / 3}}{\left(|u|+v_{d} j /\left(|u|^{3}+v_{c}^{3}\right)^{1 / 3}\right.}\right]\right. \\
& +\sqrt{3} \tan ^{-1}\left(\frac{\sqrt{3} v_{\alpha}}{v_{\alpha}-2 v_{c}}\right)-\sqrt{3} \tan ^{-1}\left(\frac{\sqrt{3}|u|}{|u|-2 v_{c}}\right) \\
& \}
\end{aligned}
$$


This differs from previous results apparently obtained by using a slow-down distribution for $v_{\perp}$ rather than $v$. which is incorrect. It should also be pointed out that there is a minor error in Gradshteyn and Ryzhik's integral table, formula (2.235). The first factor on the right-hand side should read a-1/3 instead of a-2/3. 


\section{REFERENCES}

[1] H. P. Furth, Bull. Am. Phys. Soc. 33 (1988) 1861.

[2] S. J. Zweben, Rev. Sci. Instrum. 57 (1986) 1723.

[3] D. P. Hutchinson, K. L. Vander Sluis, J. Sheffield, and D. J. Sigmar, Rev. Sci. Instrum. 56 (1985) 1075.

[4] P. P. Woskov, J. S. Machuzak, R. C. Myer, D. Y. Rhee, D. R. Cohn, N. L. Bretz, P. C. Efthimion, and J. L. Doane, Bull. Am. Phys. Soc. 33 (1988) 2098.

[5] L. Vahala, G. Vahala, and D. J. Sigmar, Nucl. Fusion 28 (1988) 1595.

[6] T. P. Hughes and S. R. P. Smith, Nucl. Fusion 28 (1988) $145 i$.

[7] I. H. Hutchinson, JET Joint Undertaking Report JET-R(87)07, Abingdon, Oxon, OX143 EA, UK.

[8] K. L. Wong and M. Ono, Nucl. Fusion 24 (1984) 615.

[9] G. Bekefi, Radiation Processes in Plasmas, John Wiley and Sons, inc. (1966). p. 128.

[10] T. H. Stix, Phys. Rev. Lett. 15 (1965) 878.

[11] G. A. Wurden, K. L. Wong, F. Skiff, and M. Ono, Phys. Rev. Lett 50 (1983) 1779.

[12] N. A. Krall and A. W. Trivelpiece, Principles of Plasma Physics, McGraw Hill, Inc. $\{1973\}$, p. 382.

[13] John D. Gaffey, Jr., J. Plasma Phys. 16 (1976) 149.

[14] L. Vahala, G. Vahala, and D. J. Sigmar, Nucl. Fusion 26 (1986) 51.

[15] K. L. Wong, Rev. Sci, Instrum. 56 (1985) 1073.

[16] I. S. Gradshteyn and 1. M. Ryzhik, Table of Integrals, Series and Products Carrected and Enlarged Edition, Academic Press (1980), p. 75. 


\section{FIGURE CAPTION}

Fig. 1. Schematic diagram of the scattering geometry. The incident beam has wave number $\vec{k}$ pointing vertically upward. The scattered beam has wave number $\vec{k}_{s}$ lying on a conical surtace, i.e., the scattered angle $\theta$ and the magnitude of $\vec{k}\left(\vec{k}=\vec{k}_{s}-\vec{k}_{i}\right)$ are held fixed. 
$=89 \times 0046$

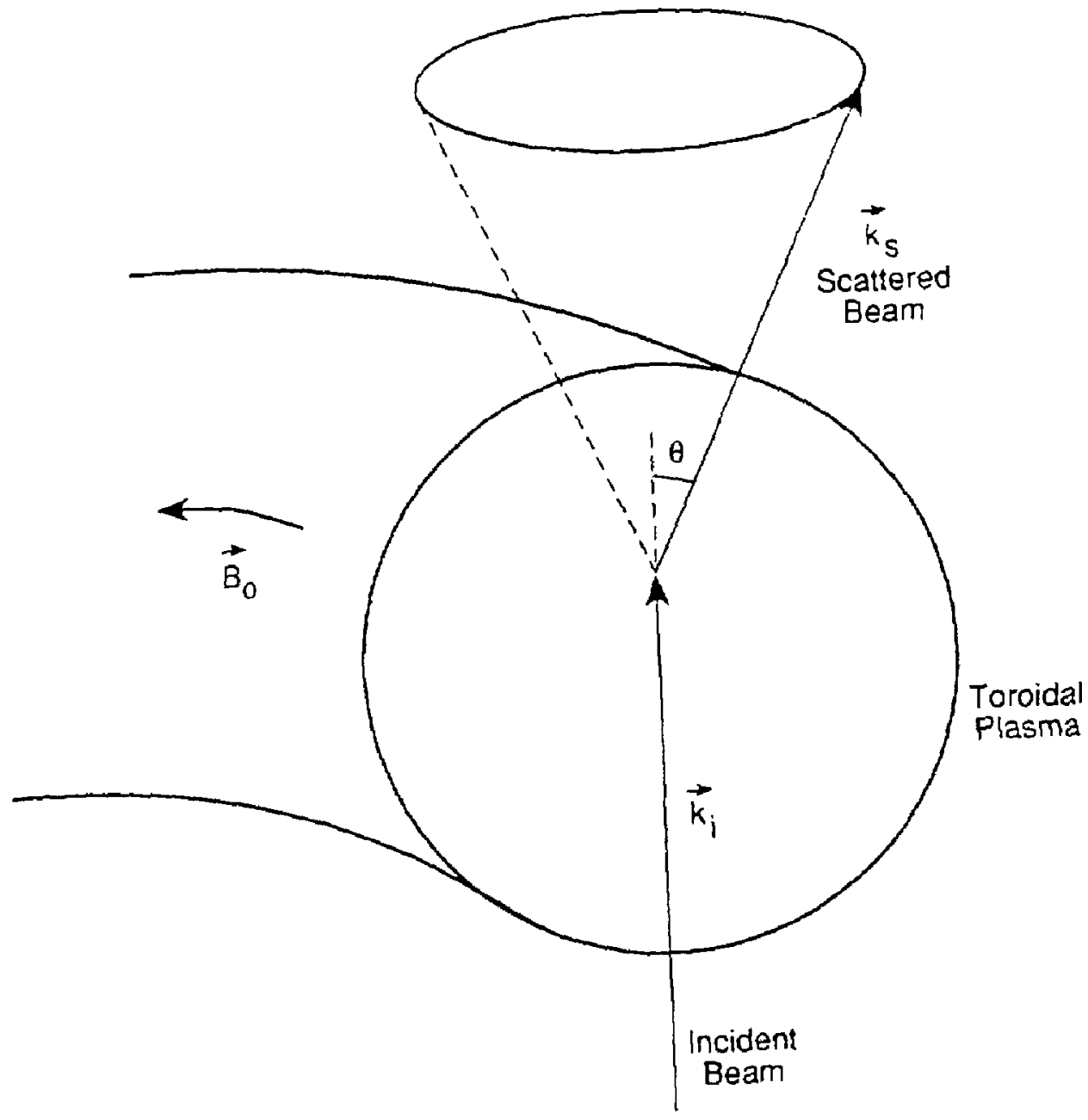

Figure I 
Or. Frank J. Pooloni, Univ of wollongong, AustRali i Prot. M.H. Bronnan, Univ Syanoy, australia

Plasmo Research Lab., Australian Nat, Univ., AUSTRALIA

Prot. I.R. Jones, Flindars univ., Australla

Prot. F. Cop, Inst Theo Phys, AUSTRIA

Prot. M. Hinder, Instut tur Theoretische Physik. AustRin

M. Coossens, Astronomisen Insti tuut, BELGIU

Ecole Royale Mititaire, Lad de Phys Plosmas, BelGium

Comerssion-Europasn, in-xil fusion Prog, BELGIUM

Prof. R. Boucique, Ai jksuniversiteit Gant, BELGIUM

Or. P.H. Soksmoka, instituto Fisica, Banzil

irstituto De Pasquisas Espaciasi-iNPE, BRAzIL

Docueants Office, atomic Endrgy of Conado Limited, Canada

Cr. M.P. Bcenynski, a Technologies, Ine., Camada

Dr. H.M. Skarsgard, University of Saskatentwan, CANADA

Dr. H. Barnard. University of British Columoia. CANAOA

Prof. J. Tuichnann, Univ. of Montreal. CANMda

Prof. S.R. Sramivasan, Univarsity of Calgary, CAMADA

Frof. Tudor W. Jahnston, IMPS-Energie, CAwan

Dr. Bolton, Contre canadien de tusion magnetique, CANAOA

Or. C.R. James, Univ. of Albarta, CAMBDA

Or. Peter Lukac, Komengkeho Univ, CZECHOSLOVAKIA

The Librarian. Culhe Laooratory. EMGLAND

The Librarian, Rutherford ADEleton Laboratory, EMGLAMD

Mrs. S.A. Huteninson, JET Librsty, England

C. Mouttet, Lab. de Paysique des Milieux lonisós, fRANCE

J. Asod, CEN/CAOARACHE - Bat 506, FRANCE

Ms. C. Rinni, Librorian, Unir, of loannina, GeECE

Or, Tom Mul, Acadeny Gibliographic Ser., Mong xang

Prepriat Library, Hungarian Aeadewy of Sciences, HuMgary

Pr. B. Jas Gudta, Sahs ingt of Nucl. Phys., InOtA

Dr. P. Kan, Institute for Plasmo Researen, INDIA

Or. Philis Rosenou, isroel inst. of Tech, 1SRAEL

Liprarian, Ine'l Cer Theo Phys, ITAly

Prof. G. Rostagni, Istituto Gas lonizzoti Jul ONR, ITALY

Miss Clelis Da Polo. Assoc EuRatow-Ehea, italr

Dr. G. Grosso, istituto di fisica del PI asto. ITALY

Dr. M. ramoto, Tosniba Res \& Oev, JaPan
Prot, 1. Mavakani, Atomic Energy Res, inst -ute, JAPan

arot. Kraj, Nismikdwa. Univ at Mirosmime, MPAN

Jirector. Dept. Lorge iokamak Res. JAER!, IAPAN

Prot, Satosni Iten, Kyushu university, JAPaN

Research Into Cantar. Nagoyd University, JAFAN

Prof. S. Tanaka. Kyoto University, JAPAN

Liorary, Kroto University, JAPAN

Prol. Nobusuk, inowe, Univers, ty of TOWYO, JAPAN

S. Nor, JAER!, JAPAN

H. Jeong, Liorarian, Korea Aovanced Enurgy Res inst, KORE:

Prot. 0.1. Choi, The Kores Adv. Inst of Sci \& Tech, KOREa

Prot. a.S. Liler, Uniwersity of Waikato. NEW ZEALANO Instipute of Plasan Physics, PEOPLE'S REPU⿴LIC OF CHINA Librarian, Instivute of Phys., PEOfLE'S REPU⿴Lit OF CHINA Library. Tsing Hua Univarsity, PEOPLE'S REPUBLIC OF CHINA

2. Li, Southwest Ingt. Physics, PEOPLE'S REPUBL IC OF CHINA Prot. J.A.C. Cabral, Inst Suderior Tecnico, PORTLGAL Or. Octavian Parrus, AL I CUza univarsipy. ROMania Dr. Jam de Villiers, Fusion Studies, AEC, SO AFPICA Prof. M.A. Heliberg. University of Natal, SO AFAICA C.I.E.M.A.T., Fusion Div. Library, SPAIN Dr. Lennart Stenflo, University of LAEA, SWEDEN library. Reyal institute of Teen. SwEDEN Prot. Hans Wilnglason, Chalmers Univ of Tech, SWEDEN Centre Phys des plasmas, Ecole Polytech Fed, SWITZERLAND Gibliothaek, Fom-Inst Voar Plasma-Fysica, He NETHERLANOS Merin Durgut, Midale East Technieal University, TURKEY Dr. D.D. Ryutor, Sibarian Acad Sci. USSR

Dr. G.A. Eliseev, Kurchator Institute, USSR Dr. V.A. Giukhikh, Inst Electrophysical Apparatus, USSR Prot. 0.5. Padichenka, Inst. of Phys. I Teeh. USSR Or. L.M. Kovrizhnykn, Institute of Gen. Physics, USSR Nuclear Res. Estadishment, dulich ltd., H. GEPaMAY Bibliotnek. Inst. Fur Plasmaforsenung, H. GERAN Dr. K. Schindler. Runr-Universitat Bochum, w. GePMany aSDEx Rosting Fin, cro wagner, IPP,Max-Disnck, GERMANr Librarian. Max-Plonck Institut, H. GEPAMAY Prot. R.K. Janev. Inst of Phys. TUGOSLAVIA 